\title{
Morphological diversity of phytolith structures in six species of Carex L. and Cyperus L. (Cyperaceae Juss.) from West Bengal, India
}

\author{
SUBRATA MAJUMDER ${ }^{1}$, TANMOY MALLICK ${ }^{1,2}$, ASOK GHOSH $^{1, \bullet}$ \\ ${ }^{1}$ Taxonomy and Biosystematics Laboratory, UGC-CAS Department of Botany (DST-FIST sponsored), The University of Burdwan, Golapbag \\ 713104, West Bengal, India. Tel.: +91-342-2656427, ’email: asokcarex@gmail.com; asokcarex@rediffmail.com \\ ${ }^{2}$ Department of Botany, Ananda Mohan College. 102/1 Raja Rammohon Sarani, Kolkata 700009, West Bengal, India
}

Manuscript received: 19 April 2020. Revision accepted: 7 July 2020.

\begin{abstract}
Majumder S, Mallick T, Ghosh A. 2020. Morphological diversity of phytolith structures in six species of Carex L. and Cyperus L. (Cyperaceae Juss.) from West Bengal, India. Biodiversitas 21: 3471-3486. Cyperaceae is a taxonomically challenging group due to its cosmopolitan distribution, similar vegetative structure, and reduced reproductive morphology. The present study focused on the characterization and description of phytolith morphotypes based on shape, structure, and ornamentation. Six species of Carex L. and Cyperus L. (three species each) were considered for the present study. From the investigation, we found 56 phytolith morphotypes, among which the principle ones were conical, elongate bulbous margin, lanceolate psilate, tabular concave with verrucate, tabular concave columellate, ovate granulate, and orbicular concave. The conical morphotype was most commonly found. The elongate bulbous margin, lanceolate psilate, and tabular concave with verrucate phytolith morphotypes were specific to the genus Carex, while the tabular concave columellate, ovate granulate and orbicular concave were only found in Cyperus spp. Further, the detailed study of conical morphotypes revealed a distinctive character among the studied genera. Our study concludes that conical, elongate bulbous margin, lanceolate psilate, tabular concave with verrucate, tabular concave columellate, ovate granulate, and orbicular concave morph otypes are constant at the genus level and may play a role in taxonomic identification in the family Cyperaceae.
\end{abstract}

Keywords: Carex, Cyperaceae, Cyperus, phytolith morphotypes, silica body

\section{INTRODUCTION}

With 98 genera and approximately 5500 species, Cyperaceae is the third largest family of monocotyledons (WCSP 2020), In India, Cyperaceae is the sixth largest family, with 38 genera and 545 species (Arisdason and Lakshminarasimhan 2017), Karthikeyan et al. (1989) reported 163 species of Carex from India and Prasad and Singh (2002) reported 70 species of Cyperus from India. Due to its cosmopolitan distribution, short life cycle pattern, similarity in vegetative morphology and highly reduced reproductive structure, the taxonomy of Cyperaceae is difficult (Reznicek and Catling 1986; Reznicek 1990; Starr et al. 1999; Simpson and Inglis 2001; Starr and Ford 2001; Pal and Choudhury 2014).

In monocotyledons, especially the members of the families Cyperaceae and Poaceae are the most important phytolith accumulators in the clade Poales (Naskar and Bera 2018; Fernández Honaine et al. 2009),

Silica deposition occurs in epidermal cells and vascular strands of the plant body (Hodson et al. 2005; Piperno 2006; Eksambekar 2009; Lisztes-szabo et al. 2015; Naskar and Bera 2018), The shape and size, ornamentation patterns, and structure of phytoliths depend upon the cell structure on which the silica bodies are deposited (Naskar and Bera 2018), The structure of phytolith may vary at the family level and in some cases at the genus or species level (McNamee 2013; Naskar and Bera 2018), The formation of phytoliths, the shape and size of morphotypes are not only controlled by environmental conditions but also by genetic influence (Prychid et al. 2004).

Fernández Honaine et al. (2009) reported that the conical silica body type with or without a variable number of satellite bodies is the dominant morphotype among different tribes of Cyperaceae. However, this conical morphotype is not unique to the family Cyperaceae and is also present in Orchidaceae and Arecaceae (Ollendorf 1992), In the case of Orchidaceae and Arecaceae, the conical silica bodies are never found in the leaf epidermis, and they never form a plate-like structure (i.e., present singly) (Ollendorf 1992), Epidermal micro-morphology of the leaf, culm, and achene was used to delimit the sections of Carex, Cyperaceae (Star and Ford 2001).

In the last few decades, phytolith morphotypes concerning systematics of different taxa of angiosperms have been evaluated by different authors in different ways. Netolitsky (1929) revised phytolith morphotypes and attempted to identify marker phytolith morphotypes in different taxa of flowering plants. Subsequently, phytolith characteristics were used effectively by several authors in the characterization and identification of different members of grasses (Rosen 1992; Wang and Lu 1993; Pearsall et al. 1995; Houyouan et al. 1997; Zhao et al. 1998; Fahmy 2008; Shakoor et al. 2014), Mudassir et al. (2018) effectively and efficiently used phytolith signatures (elemental composition) to identify three species of Setaria (Poaceae), Prychid et al. (2004) summarized the silica morphotypes in the tribe Cypereae of the subfamily Cyperoideae and in the tribe Cariceae of the subfamily Caricoideae. Though, Muasya et al. (2009) divided the 
family Cyperaceae into two subfamilies: Cyperoideae and Mapanioideae, based on molecular data.

Phytoliths were added to the taxonomy of Cyperaceae by Toivonen and Timonen (1976) and Browning and Gordon Gray (1995), The Cyperaceae family can be distinguished from other major phytolith-producing angiosperms families (except Orchidaceae and Arecaceae) based on its characteristic conical morphotype (Piperno 1988; Mulholland et al. 1989; Ollendorf 1992; Kondo et al. 1994; Wallis 2003; Fernández Honaine et al. 2009, 2013), The most recent work on sedge phytoliths was conducted by Bobrov et al. (2016), Murungi (2017), and Murungi and Bamford (2020), emphasizing the achene conical morphotype and non-conical morphotypes.

According to Fernández Honaine et al. (2013), the papillae morphotype (referred to as conical in the present treatment) found in Cyperaceae is a major morphotype, whereas the family Poaceae is characterized by the bilobate morphotype (Naskar and Bera 2018).

Piperno (1988) considered the pointed or blunted apices and smooth to nearly smooth peripheral surface as conicalshaped morphotypes, whereas Mulholland et al. (1989) categorized the conical structure into two types: one is long, sharply hollow pointed, and the other is short, solid, and resembles Hershey's kisses. Eventually, many researchers applied different terms, such as Cyperaceous type (Mehra and Sharma 1965), cones (Metcalfe 1971), conical-shaped (Piperno 1985), hat-shaped (Piperno 1988), and papillae (Stevanato et al. 2019), all of which are synonymous.

Phytolith characteristics are considered ready references for systematic treatments in different taxa of Poaceae (Twiss et al. 1969; Piperno 1998, 2006; Gallego and Distel 2004; Naskar and Bera 2018), This is possible because there is a reference standard of these morphotypes in Poaceae.

The present investigation was undertaken to study the phytolith morphotypes found in different parts of the plant and to understand the effectiveness of phytolith morphotypes for taxonomic identification and differentiation of the genera Carex and Cyperus of Cyperaceae.

\section{MATERIALS AND METHODS}

\section{Materials}

Six species of Cyperaceae were collected from different locations in the Burdwan and Darjeeling districts, West Bengal, India. For phytolith analysis, leaf blades, leaf sheaths, and culms were collected separately and dried using silica gel and brought to the laboratory for oxidation treatment. Voucher specimens are deposited in BURD (Thiers 2019), and details of the collection are given in Table 1.

\section{Methods}

Phytoliths were extracted using the method of $\mathrm{Lu}$ and Liu (2003) and Dhooge (2005) with some modifications. Five samples were considered for each studied species. First, $1 \mathrm{gm}$ of plant parts (leaf blade, leaf sheath, and culm treated separately) were soaked in distilled water overnight and rinsed with distilled water five to six times. Then, the plant parts were dried properly and treated with 1:1 sulfuric acid and nitric acid, and heated at $150^{\circ} \mathrm{C}$ for 10 to 15 minutes. Next, the mixture was centrifuged at $5000 \mathrm{rpm}$ for 5 minutes, and the pellet was collected. The centrifugation process was repeated five times to remove all the acid residues. Subsequently, the washed materials were diluted in $1 \mathrm{ml}$ of distilled water. One drop of the suspension was placed on a slide and covered with a cover glass and observed under a Magnus MLX light microscope (model no: 527955) and a Leitz LABORLUX S (model no: 512859/102299) microscope.

For confirmation, we considered a morphotype when it occurred at least five times in a particular sample. We observed all the morphotypes present in plant parts. Images were captured using a Magcam DC14 (S/N C: 1804044093), Length of the conical, height of conical, height of apex, the width of apex, and inter-apical area distance were measured from the captured images, using Digimizer software (version 4.6.1), We measured characters of conical morphotype seven to twenty-one times for a particular species. The data were used to determine the mean value with standard deviation.

The description and characterization of the phytolith morphotypes were performed following ICPN 1.0 (Madella et al. 2005) and ICPN 2.0 (Neumann et al. 2019); Fernández Honaine et al. (2009); Mercader et al. (2010); Lisztes-Szabo et al. (2014, 2015) and Biswas et al. (2016).

The detailed phytolith micromorphological study was conducted using FE-SEM analysis for two selected phytolith morphotypes (conical and elongate bulbous margin), For FE-SEM sample preparation, diluted extractions were dried in a hot-air oven for 10 minutes and then put on a carbon-coated stab. Then, the samples were gold coated for 3 minutes. High-resolution photography was performed using a ZEISS Gemini Field-emission Scanning Electron Microscope (Model no. Sigma 300), and the images were analyzed with the help of Digimizer software (version 4.6.1)

Table 1. Collection details of the studied species

\begin{tabular}{llrc}
\hline Name of the species & Collection area with coordination & Date of collection & Voucher No. \\
\hline Carex cruciata Wahlend. & Zoological park, Darjeeling $\left(27.2907^{\circ} \mathrm{N} 88.3134^{\circ} \mathrm{E}\right)$ & 25.11 .2017 & $\mathrm{BURD} 12148$ \\
Carex filicina Nees. & Gayabari, Darjeeling $\left(26.8567^{\circ} \mathrm{N} 88.3754^{\circ} \mathrm{E}\right)$ & 15.05 .2015 & $\mathrm{BURD}$ \\
Carex setigera D. Don & Garidhwa, Darjeeling $\left(27.0131^{\circ} \mathrm{N} 88.2515^{\circ} \mathrm{E}\right)$ & 16.05 .2015 & $\mathrm{BURD}$ \\
Cyperus distans L.f. & Golapbag, Burdwan $\left(23.2532^{\circ} \mathrm{N} 87.8465^{\circ} \mathrm{E}\right)$ & 25.10 .2017 & $\mathrm{BURD}$ \\
Cyperus exaltatus Retz. & Durgapur, Burdwan $\left(23.2907^{\circ} \mathrm{N} 88.3134^{\circ} \mathrm{E}\right)$ & 15.11 .2017 & $\mathrm{BURD}$ \\
Cyperus imbricatus Retz. & Golapbag, Burdwan $\left(23.2514^{\circ} \mathrm{N} 87.8460^{\circ} \mathrm{E}\right)$ & 15.09 .2017 & $\mathrm{BURD}$ \\
\hline
\end{tabular}




\section{RESULTS AND DISCUSSION}

\section{Results}

During the present investigation, 56 phytolith morphotypes were observed (Table 2; Figures 1 and 2), Among them, 15 phytolith morphotypes are common in both the genera Carex and Cyperus; 26 morphotypes are specific to the genus Carex (Table 3) and 15 morphotypes are specific to Cyperus (Table 3), whereas 27 morphotypes are unclassified. Of the studied morphotypes, the most common are conical (observed in all the studied species), elongate bulbous margin, lanceolate psilate, tabular concave with verrucate, tabular concave columellate, ovate granulate, orbicular concave, favose, elongate form, stomatal complex, scutiform, tabular form, tower, and tower wide. Details of the morphotypes (plant part wise) and descriptions are provided in Table 2 and Figures 1 and 2. FE-SEM analyses of two selected phytolith morphotypes are presented in Figures 3 and 4.

In Carex, elongate bulbous margin, trapezoid, tabular concave with verrucate morphotypes are found in leaf blades and leaf sheath. Whereas elongate laminate found in culm and lanceolate form is found in leaf blade only (Table 2, Figure 1).

In Cyperus, orbicular concave morphotype found in culm, tabular concave columellate morphotype found in leaf blade and ovate granulate morphotype found in both leaf blade and leaf sheath (Table 2, Figure 2).

Though, tower wide and conical morphotypes are found in both leaf blade and leaf sheath, and stomatal complex, favose are found only in leaf blade, whereas tower morphotype is found in leaf sheath of all the studied species (Table 2).

Elongate bulbous morphotype (characterized by rectangular shape with two times longer arms than width, margin with series of balloon or bulging like structure) found only in studied species of Carex. In C. cruciata base or stalk of the bulbous structure is narrow to short and the surface is smooth (Figure 3.A), Besides this, C. filicina and C. setigera shape of the bulbous structure is quite similar; though, in $C$. setigera the stalk is broad and short, and the bulbous surface is wavier (Figure 3.B, C).

Among the characters of the conical phytolith, margin of conical, form of conical, arrangement of conical (platelet/individual), apex structure, apex format, the arrangement of peripheral satellite, and inter apical area (zone) with or without satellite are also presented in Table 4 and Figure 5 as qualitative variations of the conical morphotype.

Silica bodies are present singly in several bodies per unit (Figures 1 and 2), The variation in the structures is mainly found in the number of peripheral satellites (Figure 3 ), These small cones or satellites around the main conical body are variable from species to species.

In all three studied species of Carex, the conical length was more than $11 \mu \mathrm{m}$ (Figures 5.C, E; Figure 6), while in the three studied Cyperus species, the conical length was less than $11 \mu \mathrm{m}$ (Figures 5.C, E; Figure 6), The height of the conical morphotypes in Carex species was more than 6 $\mu \mathrm{m}$ (Figures 5.C and 6), while it was less than $6 \mu \mathrm{m}$ in species of Cyperus (Figures 5.C and 6), Similarly, the apex width in species of Carex was more than $5 \mu \mathrm{m}$ and in Cyperus was less than $5 \mu \mathrm{m}$. The apex height in species of Carex was more than $3 \mu \mathrm{m}$, while that in Cyperus species was less than $3 \mu \mathrm{m}$ (Figures 5 and 6).

\section{Discussion}

Among the 56 phytolith morphotypes, elongate morphotypes are found in all the studied Carex and Cyperus species, and the morphotypes were previously recorded by Fernández Honaine et al. (2009) and Stevanato et al. (2019) from different species of the genera Carex and Cyperus of Cyperaceae. Elongate forms of morphotypes were also described by Twiss et al. (1969), and Lisztesszabo et al. (2015) in Poaceae; Prychid et al. (2004) in Orchidaceae; Ebigwai et al. (2015) in Cucurbitaceae; Collura and Neumann (2017) in Anacardiaceae and Sapotaceae.

The elongate bulbous margin phytolith morphotype was found in species of Carex (C. cruciata, C. filicina, $C$. setigera) (Figures 1.A-E; 4.A-C), Previously, Murungi (2017) described elongate crenate or psilate morphotype from the leaf of Bulbostylis, Fuirena, and Scleria, and also reported that elongate crenate plate as the most dominant phytolith morphotype in $F$. pubescens.

Meanwhile, tabular (Fernández Honaine et al. 2009; Mercader et al. 2010) phytolith morphotypes were found in all the studied species, and the morphological variation was low for genus-level identification.

The term tower phytolith morphotype was first introduced by Lu and Liu (2003), Subsequently, tower wide morphotypes were reported by Mercader et al. (2010), In the present study, a tower form of phytolith morphotypes [Like- tower (Figures 1.AQ, AR; 2.Z), tower wide (Figures 1.BB; 2.AG, AH)], were found in almost all the studied species of Carex and Cyperus.

From a comprehensive observation of the studied species of Cyperus and Carex, Ollendorf (1992) found a rounded, individual, psilate cone base with a pointed apex, without peripheral satellites. With the similar objective of Ollendorf (1992), Fernández Honaine et al. (2009) and Stevanato et al. (2019) reported conical or papillae morphotypes in fruits and leaf respectively. Variations were also described in ornamentation, apex format, base form, and base shape of conical (polygonal in fruit; square, rectangular, rounded, hexagonal, oblong in leaf), The present results are similar to those of Ollendorf (1992), except for the arrangement of the satellites and conical. This study clarified and improved the characterization of conical structures. Here, conicals are arranged in a platelet form, and satellites are surrounding the central apex with an oblong conical base shape (Figures 3 and 5), The studied species showed two distinct sub-morphotypes: (i) the oblong-shaped conical base is found in all three species of Carex (Figures 3 and 5.B), and (ii) the square-shaped conical base is evident in all the studied species of Cyperus (Figures 3 and 5.A), 
Table 2. Phytolith morphotypes present in different parts of the investigated species of Cyperaceae and their descriptions (new phytolith descriptors marked in bold)

\begin{tabular}{|c|c|c|c|c|}
\hline First descriptor & Second descriptor & Studied species & $\begin{array}{l}\text { Studied plant } \\
\text { parts }\end{array}$ & Description of morphotypes \\
\hline $\begin{array}{l}\text { Elongate (Twiss et al. } \\
\text { 1969) }\end{array}$ & Bulbous margin (this study) & 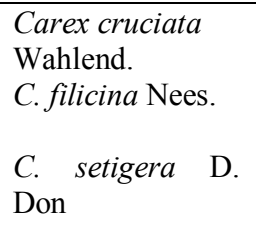 & $\begin{array}{l}\text { Leaf blade } \\
\text { Leaf sheath } \\
\text { Leaf blade } \\
\text { Leaf sheath } \\
\text { Leaf blade }\end{array}$ & Much longer than wide; margin with globular or enlarge bulb. (Figure 1.A-E) \\
\hline $\begin{array}{l}\text { Stomatal complex } \\
\text { (Carnelli et al. 2004) }\end{array}$ & & $\begin{array}{l}\text { C. cruciata } \\
\text { C. filicina } \\
\text { C. setigera }\end{array}$ & $\begin{array}{l}\text { Leaf blade } \\
\text { Leaf blade } \\
\text { Leaf blade }\end{array}$ & Stomata with guard cell. (Figure 1.F-H) \\
\hline $\begin{array}{l}\text { Elongate (Twiss et al. } \\
\text { 1969) }\end{array}$ & Articulated (ICPN 2.0) & C. cruciata & Leaf blade & Much longer than wide, jointed, or attached. (Figure 1.I) \\
\hline Trapezoid (ICPN 2.0) & Sinuate (ICPN 2.0) & C. cruciata & Leaf blade & $\begin{array}{l}\text { Having the outline of a trapeziform, with four unequal sides, none of them parallel, and } \\
\text { margins with uneven concavities and convexities. (Figure 1.J) }\end{array}$ \\
\hline Trapezoid (ICPN 2.0) & & C. cruciata & Leaf sheath & $\begin{array}{l}\text { Having the outline of a trapeziform, with four unequal sides, none of which are parallel. } \\
\text { (Figure } 1 . \mathrm{K} \text { ) }\end{array}$ \\
\hline Lanceolate (ICPN 1.0) & Psilate (ICPN 2.0) & $\begin{array}{l}\text { C. cruciata } \\
\text { C. filicina }\end{array}$ & Leaf blade & Shape like a lance head, longer than wide, with a broad base and blunted tip. (Figure 1.L) \\
\hline Lanceolate (ICPN 1.0) & Reflexed (ICPN 1.0) & C. setigera & Leaf blade & $\begin{array}{l}\text { Lance head-like body; longer than wide, with a broader base and slightly curved. (Figure } \\
\text { 3.M) }\end{array}$ \\
\hline $\begin{array}{l}\text { Elongate (Twiss et al. } \\
\text { 1969) }\end{array}$ & Papillate (ICPN 2.0) & C. cruciata & $\begin{array}{l}\text { Leaf blade } \\
\text { Leaf sheath }\end{array}$ & Much longer than wide; surface with minute or acute protuberances. (Figure 1.N, O) \\
\hline Rondel (ICPN 2.0) & Tenuis reflexed (this study) & $\begin{array}{l}\text { C. cruciata } \\
\text { C. filicina }\end{array}$ & $\begin{array}{l}\text { Leaf blade } \\
\text { Leaf sheath }\end{array}$ & $\begin{array}{l}\text { They show rounded or intermediate forms in the top and bottom view and concave in } \\
\text { the side view. (Figure 1.P, Q) }\end{array}$ \\
\hline $\begin{array}{l}\text { Elongate (Twiss et al. } \\
\text { 1969) }\end{array}$ & Psilate (ICPN 2.0) & C. cruciata & Leaf blade & Much longer than wide with a smooth surface. (Figure 1.R) \\
\hline Favose (ICPN 1.0) & & $\begin{array}{l}\text { C. cruciata } \\
\text { C. filicina }\end{array}$ & $\begin{array}{l}\text { Leaf blade } \\
\text { Leaf blade }\end{array}$ & Honeycombed structure, arranged in a parallel form. (Figure 1.S) \\
\hline Conical (Ollendorf 1992) & & $\begin{array}{l}\text { C. cruciata } \\
\text { C. filicina } \\
\text { C. setigera }\end{array}$ & $\begin{array}{l}\text { Leaf blade } \\
\text { Leaf sheath } \\
\text { Leaf blade } \\
\text { Leaf sheath } \\
\text { Leaf blade } \\
\text { Leaf sheath }\end{array}$ & $\begin{array}{l}\text { Cone-shaped structure, base, and cones are not differentiated, and the tips of the cones are } \\
\text { pointed. (Figure 1.T-Y) }\end{array}$ \\
\hline $\begin{array}{l}\text { Elongate (Twiss et al. } \\
\text { 1969) }\end{array}$ & Lanceolate (ICPN 1.0) & C. cruciata & Leaf sheath & Much longer than wide; lance-shaped head base is broader than the apex. (Figure 1.Z) \\
\hline Rectangle (ICPN 1.0) & Psilate (ICPN 2.0) & & & $\begin{array}{l}\text { Having four sides more or less at a } 90^{\circ} \text { angle, each side with the same length as the one } \\
\text { opposite, with a smooth surface. (Figure 1.AA) }\end{array}$ \\
\hline
\end{tabular}




\begin{tabular}{|c|c|c|c|c|}
\hline Scutiform (ICPN 1.0) & Triangular (this study) & $\begin{array}{l}\text { C. cruciata } \\
\text { C. setigera }\end{array}$ & $\begin{array}{l}\text { Leaf sheath } \\
\text { Leaf sheath }\end{array}$ & Shield-shaped structure, tri-angled. (Figure 1.AB, AC) \\
\hline Tabular (ICPN 2.0) & Scrobiculate (ICPN 2.0) & C. cruciata & Culm & Table-like flat pitted surface. (Figure 1.AD) \\
\hline $\begin{array}{l}\text { Elongate (Twiss et al. } \\
\text { 1969) }\end{array}$ & Sinuate (ICPN 2.0) & C. cruciata & Culm & $\begin{array}{l}\text { Much longer than wide, having a margin with alternating but uneven concavities and } \\
\text { convexities. (Figure 1.AE) }\end{array}$ \\
\hline Tracheary (ICPN 2.0) & & $\begin{array}{l}\text { C. cruciata } \\
\text { C. filicina } \\
\text { C. setigera }\end{array}$ & $\begin{array}{l}\text { Culm } \\
\text { Leaf blade } \\
\text { Leaf blade }\end{array}$ & $\begin{array}{l}\text { Cylindrical and elongate bodies, relatively straight surface covered with ring to helical- } \\
\text { shaped ridges arranged perpendicular to the long axis. (Figure } 1 . \mathrm{AF}, \mathrm{AG} \text { ) }\end{array}$ \\
\hline Tabular (ICPN 2.0) & Psilate (ICPN 2.0) & C. cruciata & Culm & Table-like surface, flat and smooth. (Figure 1.AH) \\
\hline $\begin{array}{l}\text { Elongate (Twiss et al. } \\
\text { 1969) }\end{array}$ & $\begin{array}{l}\text { Concave with verrucate (this } \\
\text { study) }\end{array}$ & C. filicina & Leaf blade & $\begin{array}{l}\text { Much longer than wide, both ends are concave with irregularly shaped, wart-like } \\
\text { processes. (Figure 1.AI) }\end{array}$ \\
\hline $\begin{array}{l}\text { Acute bulbosus (ICPN } \\
2.0)\end{array}$ & & C. filicina & Leaf blade & Solid-body with a narrower apex and a spherical to fusiform-shaped base. (Figure 1.AJ) \\
\hline $\begin{array}{l}\text { Trichome (Carnelli et al. } \\
\text { 2004) }\end{array}$ & & C. filicina & Leaf blade & Cylindrical body with a wide base and a smooth surface. (Figure 1.AK) \\
\hline $\begin{array}{l}\text { Crescenti (Twiss et al. } \\
\text { 1969) }\end{array}$ & & C. filicina & Leaf sheath & A crescent-shaped structure with a smooth surface. (Figure 1.AL) \\
\hline Tabular (ICPN 2.0) & Gibbous (ICPN 1.0) & C. filicina & Leaf sheath & Elongated, and the margins are very convex. (Figure 1.AM) \\
\hline $\begin{array}{l}\text { Elongate (Twiss et al. } \\
\text { 1969) }\end{array}$ & Echinate (ICPN 2.0) & C. filicina & Leaf sheath & Much longer than wide and margins with prickles. (Figure 1.AN) \\
\hline $\begin{array}{l}\text { Crescenti (Twiss et al. } \\
\text { 1969) }\end{array}$ & Convex (ICPN 2.0) & C. filicina & Leaf sheath & $\begin{array}{l}\text { A crescent-form structure with a smooth surface and a deeply convex inner portion. (Figure } \\
\text { 1.AO) }\end{array}$ \\
\hline Cuneiform (ICPN 1.0) & & C. filicina & Leaf sheath & Wedge-shaped bulliform cell. (Figure 1.AP) \\
\hline Tower (Lu and Liu 2003) & & $\begin{array}{l}\text { C. filicina } \\
\text { C. setigera }\end{array}$ & $\begin{array}{l}\text { Leaf sheath } \\
\text { Leaf sheath }\end{array}$ & Conical tall body with a flat base and flat apex. (Figure 1.AQ, AR) \\
\hline Tabular (ICPN 2.0) & Sinuate (ICPN 2.0) & $\begin{array}{l}\text { C. filicina } \\
\text { C. setigera }\end{array}$ & $\begin{array}{l}\text { Culm } \\
\text { Leaf blade } \\
\text { Leaf sheath }\end{array}$ & $\begin{array}{l}\text { Elongate tabular shaped, margins with uneven concavities and convexities. (Figure 1.AS, } \\
\text { AT) }\end{array}$ \\
\hline $\begin{array}{l}\text { Elongate (Twiss et al. } \\
\text { 1969) }\end{array}$ & Laminate (ICPN 1.0) & $\begin{array}{l}\text { C. filicina } \\
\text { C. setigera }\end{array}$ & $\begin{array}{l}\text { Culm } \\
\text { Culm }\end{array}$ & Much longer than wide, and the surface is covered with layers. (Figure 1.AU) \\
\hline Tabular (ICPN 2.0) & $\begin{array}{l}\text { Concave echinate with } \\
\text { stomata (this study) }\end{array}$ & C. setigera & Leaf blade & $\begin{array}{l}\text { Elongated structure and both ends are curved inward; stomata are attached to this } \\
\text { curved portion. (Figure 1.AV) }\end{array}$ \\
\hline Tabular (ICPN 2.0) & $\begin{array}{l}\text { Concave echinate (this } \\
\text { study) }\end{array}$ & C. setigera & Leaf blade & Elongate tabular body with curved ends; surface with prickles. (Figure 1.AW) \\
\hline Tabular (ICPN 2.0) & $\begin{array}{l}\text { Concave with verrucate (this } \\
\text { study) }\end{array}$ & $\begin{array}{l}\text { C. cruciata } \\
\text { C. filicina } \\
\text { C. setigera }\end{array}$ & $\begin{array}{l}\text { Leaf blade } \\
\text { Leaf sheath } \\
\text { Leaf blade }\end{array}$ & Elongate tabular body with curved ends; surface with prickles. (Figure 1.AX) \\
\hline Tabular (ICPN 2.0) & Concave (ICPN 2.0) & C. setigera & Leaf blade & Elongate tabular body with curved ends. (Figure 1.AY) \\
\hline Semi square (this study) & Sinuate (ICPN 2.0) & C. setigera & Leaf blade & $\begin{array}{l}\text { More or less square-shaped structure, with four arms; margin with uneven concavities } \\
\text { or convexities. (Figure 1.AZ) }\end{array}$ \\
\hline Tabular (ICPN 2.0) & Columellate (ICPN 1.0) & C. setigera & Leaf blade & Elongate bodies with straight rod- or pillar-like projections are found. (Figure 1.BA) \\
\hline $\begin{array}{l}\text { Tower wide (Mercader et } \\
\text { al. 2010) }\end{array}$ & & C. setigera & Leaf blade & Cone-shaped tall body with a flat apex and a wider base than apex. (Figure 1.BB) \\
\hline
\end{tabular}




\begin{tabular}{|c|c|c|c|c|}
\hline Tabular (ICPN 2.0) & Favose castelate (ICPN 1.0) & C. setigera & Leaf blade & $\begin{array}{l}\text { Elongate, honeycomb-like ornamentation and margins with square or rectangular processes. } \\
\text { (Figure 1.BC) }\end{array}$ \\
\hline Tabular (ICPN 2.0) & Crenate (ICPN 2.0) & C. setigera & Leaf blade & Elongate body, dented, with teeth-like margins. (Figure 1.BD) \\
\hline Clavate (ICPN 2.0) & & C. setigera & Culm & Club-shaped structure and slender towards the base. (Figure 1.BE) \\
\hline Acuminate (ICPN 1.0) & Hollow (this study) & C. setigera & Culm & Taper-pointed, hollow cylindrical body. (Figure 1.BF) \\
\hline \multirow[t]{19}{*}{ Unclassified } & & C. cruciata & Leaf blade & \\
\hline & & & Leafs & $\begin{array}{l}\text { Cone-shaped structure, wider at the base and tapering to the apex. (Figure 1.BH) } \\
\text { Conical tall bodv in which the anex ends with two horn-like outorowths. (Figure 1BD }\end{array}$ \\
\hline & & & $\begin{array}{l}\text { Leat sheath } \\
\text { Culm }\end{array}$ & $\begin{array}{l}\text { Conical, tall body in which the apex ends with two horn-like outgrowths. (Figure 1.BI) } \\
\text { Shield-shaped structure with a smooth surface. (Figure 1.BJ) }\end{array}$ \\
\hline & & & & Shield-shaped structure with wavy margins. (Figure 1.BK) \\
\hline & & C. filicina & Leaf blade & Shield-shaped structure with one end wider than the other. (Figure 1.BL) \\
\hline & & C. setigera & Leaf blade & Shield-like structure with one end wider than the other. (Figure 1.BM) \\
\hline & & C. cruciata & Culm & More or less circular to ovulate body with articulation. (Figure 1.BN-BQ) \\
\hline & & C. filicina & $\begin{array}{l}\text { Leaf blade } \\
\text { Culm }\end{array}$ & \\
\hline & & C. setigera & Leaf blade & \\
\hline & & C. cruciata & Culm & Star-shaped structure with five arms. (Figure 1.BR) \\
\hline & & C. filicina & Leaf blade & Bubble-shaped cell with a truncated appearance. (Figure 1.BS) \\
\hline & & & & $\begin{array}{l}\text { Crescent-form structure with a smooth surface and deeply convex inner portion. (Figure } \\
\text { 1.BT) }\end{array}$ \\
\hline & & & & Longer than wide, both margins acute, and concave elongated ends. (Figure 1.BU) \\
\hline & & & & Elongate; having minute rounded papillae or acute protuberances. (Figure 1.BV) \\
\hline & & & & Table-shaped structure, and one end curves inward. (Figure 1.BW) \\
\hline & & & & Triangular base and more or less tapering apex. (Figure 1.BX) \\
\hline & & & & Cone-shaped structure, which is wider at the base and tapering to the apex. (Figure 1.BY) \\
\hline & & & Culm & Conical tall with a curved cylindrical body. (Figure 1.BZ) \\
\hline & & C. setigera & Leaf blade & Kidney-shaped structure with a smooth surface. (Figure 1.CA) \\
\hline $\begin{array}{l}\text { Elongate (Twiss et al. } \\
\text { 1969) }\end{array}$ & Sinuate (ICPN 2.0) & $\begin{array}{l}\text { Cyperus distans } \\
\text { L.f. }\end{array}$ & Leaf blade & $\begin{array}{l}\text { Much longer than wide form with inclusions; one side with alternating but uneven } \\
\text { concavities and convexities. (Figure 2.A) }\end{array}$ \\
\hline Tabular (ICPN 2.0) & $\begin{array}{l}\text { Concave columellate (this } \\
\text { study) }\end{array}$ & $\begin{array}{l}\text { C. distans } \\
\text { C. imbricatus } \\
\text { Retz. }\end{array}$ & Leaf blade & $\begin{array}{l}\text { Table-like surface; the ends of the long cells are concave, and the outer surface has rod } \\
\text { or pillar-like processes. (Figure } 2 . B \text { ) }\end{array}$ \\
\hline $\begin{array}{l}\text { Elongate (Twiss et al. } \\
\text { 1969) }\end{array}$ & Echinate (ICPN 2.0) & C. distans & Leaf blade & $\begin{array}{l}\text { Much longer than wide, sometimes with inclusions; both sides beset with prickles, although } \\
\text { one side is more frequently arranged than the other side. (Figure 2.C) }\end{array}$ \\
\hline \multirow[t]{5}{*}{ Conical (Ollendorf 1992) } & & C. distans & $\begin{array}{l}\text { Leaf blade } \\
\text { Leaf sheath }\end{array}$ & Cones have a much thicker rounded base, and the tips are pointed. (Figure 2.D-I) \\
\hline & & C. exaltatus Retz. & Leaf blade & \\
\hline & & & Leaf sheath & \\
\hline & & C. imbricatus & Leaf blade & \\
\hline & Acute (ICPN 1.0) & C. distans & $\begin{array}{l}\text { Leaf sheath } \\
\text { Leaf blade }\end{array}$ & Much longer than wide, sharply pointed, and terminating very quickly. (Figure 2.J) \\
\hline 1969) & Acute (ICTIN 1.0) & C. Gosurns & Lear viduc & (V) \\
\hline Tabular (ICPN 2.0) & $\begin{array}{l}\text { Concave echinate (this } \\
\text { study) }\end{array}$ & C. distans & Leaf blade & $\begin{array}{l}\text { Table-like surface; the ends of the long cells are concave, and the margins are sinuate } \\
\text { or echinate. (Figure } 2 . \mathrm{K} \text { ) }\end{array}$ \\
\hline
\end{tabular}




\begin{tabular}{|c|c|c|c|c|}
\hline Tabular (ICPN 2.0) & Concave acute (this study) & C. distans & Leaf blade & $\begin{array}{l}\text { Table-like surface; both margins are acute, and the elongated ends are concave. } \\
\text { (Figure 2.L) }\end{array}$ \\
\hline Tabular (ICPN 2.0) & & C. distans & $\begin{array}{l}\text { Leaf blade } \\
\text { Culm }\end{array}$ & Elongate with a flat table-like surface. (Figure 2.M, N) \\
\hline & & C. exaltatus & Culm & \\
\hline Tabular (ICPN 2.0) & Papillate (ICPN 2.0) & $\begin{array}{l}\text { C. distans } \\
\text { C. exaltatus }\end{array}$ & $\begin{array}{l}\text { Leaf blade } \\
\text { Leaf blade }\end{array}$ & $\begin{array}{l}\text { Having a flat, table-like surface with sharp acute or minute rounded margin papillate. } \\
\text { (Figure } 2 . \mathrm{O}, \mathrm{P} \text { ) }\end{array}$ \\
\hline Ovate (ICPN 2.0) & Granulate (ICPN 2.0) & $\begin{array}{l}\text { C. distans } \\
\text { C. exaltatus } \\
\text { C. imbricatus }\end{array}$ & $\begin{array}{l}\text { Leaf blade } \\
\text { Leaf blade } \\
\text { Leaf sheath }\end{array}$ & Oblong but broader at one base; surface with granules. (Figure 2.Q) \\
\hline $\begin{array}{l}\text { Elongate (Twiss et al. } \\
\text { 1969) }\end{array}$ & Psilate (ICPN 2.0) & $\begin{array}{l}\text { C. distans } \\
\text { C. distans } \\
\text { C. imbricatus } \\
\text { C. distans } \\
\text { C. imbricatus } \\
\text { C. exaltatus }\end{array}$ & $\begin{array}{l}\text { Leaf blade } \\
\text { Leaf sheath } \\
\text { Leaf sheath } \\
\text { Culm } \\
\text { Culm } \\
\text { Leaf blade }\end{array}$ & Much longer than wide with a smooth surface and margins. (Figure 2.R-U) \\
\hline Tabular (ICPN 2.0) & Rectangular (ICPN 2.0) & C. distans & Leaf blade & Elongate, having four sides with $90^{\circ}$ angles, and each side has the same length. (Figure 2.V) \\
\hline Scutiform (ICPN 1.0) & Triangular (this study) & C. distans & Leaf sheath & Shield-shaped structure, tri-angled. (Figure 2.W) \\
\hline Tabular (ICPN 2.0) & Crenate (ICPN 2.0) & C. distans & Leaf sheath & Table-like surface, notched or scalloped; dented, with rounded to flat teeth. (Figure 2.X) \\
\hline Tabular (ICPN 2.0) & Crenate (ICPN 2.0) & C. imbricatus & Leaf blade & Table-like surface, notched or scalloped; dented, with rounded to flat teeth. (Figure 2.Y) \\
\hline Tower (Lu and Liu 2003) & & C. distans & Leaf sheath & Cone-shaped, wide at the base, and tapering or slender to the apex. (Figure $2 . Z$ ) \\
\hline Tracheary (ICPN 2.0) & & $\begin{array}{l}\text { C. distans } \\
\text { C. exaltatus }\end{array}$ & $\begin{array}{l}\text { Culm } \\
\text { Leaf blade } \\
\text { Culm }\end{array}$ & $\begin{array}{l}\text { Cylindrical and elongate bodies with a relatively straight surface covered with ring- to } \\
\text { helical-shaped ridges arranged perpendicular to the long axis. (Figure } 2 . \mathrm{AA}-\mathrm{AD} \text { ) }\end{array}$ \\
\hline Tabular (ICPN 2.0) & $\begin{array}{l}\text { Echinate verrucate (this } \\
\text { study) }\end{array}$ & $\begin{array}{l}\text { C. imbricatus } \\
\text { C. exaltatus }\end{array}$ & $\begin{array}{l}\text { Culm } \\
\text { Leaf blade }\end{array}$ & $\begin{array}{l}\text { Elongated and beset with prickles in one margin, while the other has irregularly } \\
\text { shaped wart-like processes. (Figure 2.AE) }\end{array}$ \\
\hline Favose (ICPN 1.0) & & C. exaltatus & Leaf blade & Honeycomb-like structure; parallel arrangement. (Figure 2.AF) \\
\hline $\begin{array}{l}\text { Tower wide (Mercader at } \\
\text { al. 2010) }\end{array}$ & & $\begin{array}{l}\text { C. exaltatus } \\
\text { C. imbricatus }\end{array}$ & $\begin{array}{l}\text { Leaf blade } \\
\text { Leaf sheath }\end{array}$ & $\begin{array}{l}\text { Cone-shaped tall body with flat apex and the base is much wider than the apex. (Figure } \\
\text { 2.AG, AH) }\end{array}$ \\
\hline $\begin{array}{l}\text { Elongate (Twiss et al. } \\
\text { 1969) }\end{array}$ & Sulcate (ICPN 1.0) & C. exaltatus & Leaf blade & Body is much longer than wide, and the surface is furrowed. (Figure 2.AI) \\
\hline Square (ICPN 1.0) & & $\begin{array}{l}\text { C. exaltatus } \\
\text { C. imbricatus }\end{array}$ & $\begin{array}{l}\text { Leaf blade } \\
\text { Culm }\end{array}$ & Having more or less four sides with $90^{\circ}$ angles. (Figure $2 . \mathrm{AJ}, \mathrm{AK}$ ) \\
\hline Tabular (ICPN 2.0) & Sinuate (ICPN 2.0) & C. exaltatus & Leaf blade & $\begin{array}{l}\text { Elongated body that is much longer than wide; margins with alternating but uneven } \\
\text { concavities and convexities. (Figure 2.AL) }\end{array}$ \\
\hline Cuneiform (ICPN 1.0) & & C. exaltatus & Leaf blade & Wedge-shaped or fan-shaped. (Figure 2.AM) \\
\hline Tabular (ICPN 2.0) & Scrobiculate (ICPN 2.0) & C. exaltatus & Leaf blade & Elongate body with a pitted surface. (Figure 2.AN) \\
\hline Short saddle (ICPN 2.0) & & C. exaltatus & Leaf blade & Smooth surface with depressed double edges. (Figure 2.AO) \\
\hline Orbicular (ICPN 1.0) & Concave (ICPN 2.0) & $\begin{array}{l}\text { C. distans } \\
\text { C. exaltatus } \\
\text { C. imbricatus }\end{array}$ & Culm & Circular body and the surface is curved inwardly in the middle. (Figure 2. AP) \\
\hline
\end{tabular}




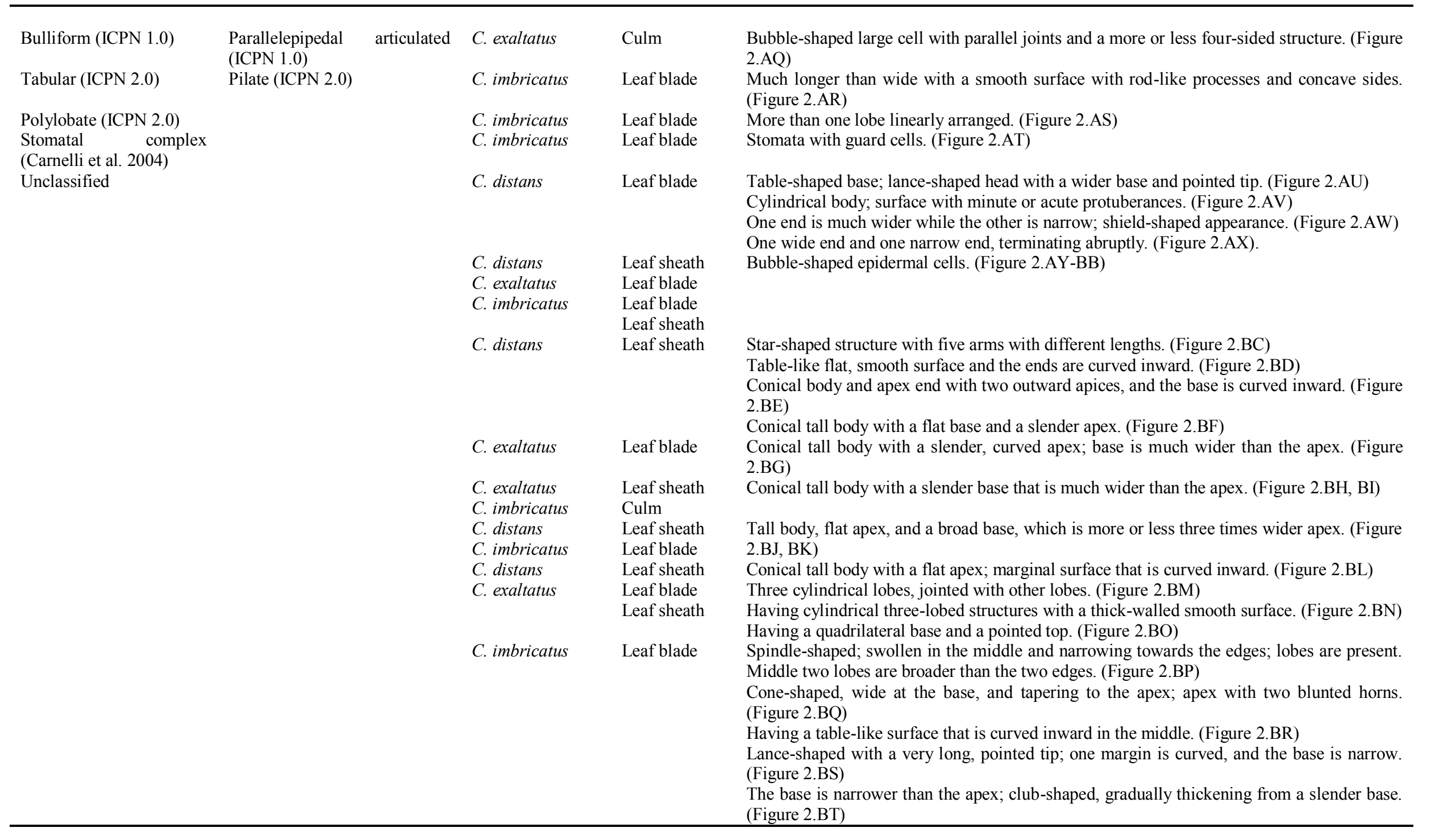


Table 3. Unique and common phytolith morphotypes found in the studied species of Carex and Cyperus at the genus level, including their count and specificity.

\begin{tabular}{|c|c|c|c|}
\hline $\begin{array}{l}\text { Number of } \\
\text { observed phytolith } \\
\text { morphotypes }\end{array}$ & $\begin{array}{l}\text { Phytolith morphotypes observed in } \\
\text { all the three studied species of } \text { Carex }\end{array}$ & $\begin{array}{l}\text { Phytolith morphotypes observed in } \\
\text { all the three studied species of } \\
\text { Cyperus }\end{array}$ & $\begin{array}{l}\text { Phytolith morphotypes } \\
\text { common in both Carex } \\
\text { and Cyperus }\end{array}$ \\
\hline 1 & Acute bulbosus & Bulliform parallelepipedal articulated & Conical \\
\hline 2 & Acuminate hollow & Elongate acute & Cuneiform \\
\hline 3 & Clavate & Elongate sulcate & Elongate echinate \\
\hline 4 & Crescenti & Polylobate & Elongate psilate \\
\hline 5 & Crescenti convex & Orbicular concave & Elongate sinuate \\
\hline 6 & Elongate articulated & Ovate granulate & Favose \\
\hline 7 & Elongate bulbous margin & Short saddle & Scutiform triangular \\
\hline 8 & Elongate concave with verrucate & Square & Stomatal complex \\
\hline 9 & Elongate laminate & Tabular & Tabular concave echinate \\
\hline 10 & Elongate lanceolate & Tabular concave acute & Tabular crenate \\
\hline 11 & Elongate papillate & Tabular concave columellate & Tabular scrobiculate \\
\hline 12 & Lanceolate psilate & Tabular echinate verrucate & Tabular sinuate \\
\hline 13 & Lanceolate reflexed & Tabular papillate & Tower \\
\hline 14 & Rectangle psilate & Tabular pilate & Tower wide \\
\hline 15 & Rondel tenuis reflexed & Tabular rectangular & Tracheary \\
\hline 16 & Semi square sinuate & & \\
\hline 17 & Tabular columellate & & \\
\hline 18 & Tabular concave & & \\
\hline 19 & Tabular concave echinate with stomata & & \\
\hline 20 & Tabular concave with verrucate & & \\
\hline 21 & Tabular favose castelate & & \\
\hline 22 & Tabular gibbous & & \\
\hline 23 & Tabular psilate & & \\
\hline 24 & Trapezoid & & \\
\hline 25 & Trapezoid sinuate & & \\
\hline 26 & Trichome & & \\
\hline
\end{tabular}

Table 4. Qualitative data of conical morphotypes (secondary descriptor) in the studied Carex and Cyperus species

\begin{tabular}{|c|c|c|c|c|c|c|}
\hline Conical character & $\begin{array}{l}\text { Carex } \\
\text { cruciata }\end{array}$ & $\begin{array}{l}\text { Carex } \\
\text { filicina }\end{array}$ & $\begin{array}{l}\text { Carex } \\
\text { setigera }\end{array}$ & $\begin{array}{l}\text { Cyperus } \\
\text { distans }\end{array}$ & $\begin{array}{l}\text { Cyperus } \\
\text { exaltatus }\end{array}$ & $\begin{array}{l}\text { Cyperus } \\
\text { imbricatus }\end{array}$ \\
\hline Shape of conical & Oblong & Oblong & Oblong & Square & Square & Square \\
\hline Margin of conical (entire/undulate) & Entire & Entire & Entire & Entire & Entire & Entire \\
\hline Form of conical (concave/convex) & Convex & Convex & Convex & Convex & Convex & Convex \\
\hline $\begin{array}{l}\text { Arrangement form of conical } \\
\text { (platelet/individual) }\end{array}$ & Platelet & Platelet & Platelet & Platelet & Platelet & Platelet \\
\hline Apex structure & Straight & Straight & Straight & Straight & Straight & Straight \\
\hline Apex format & Acuminate & Acuminate & Acuminate & Acuminate & Acuminate & Acuminate \\
\hline $\begin{array}{l}\text { Arrangement form of peripheral } \\
\text { satellite (continuous/discontinues) }\end{array}$ & Discontinues & Discontinues & Discontinues & Discontinues & Continues & Continues \\
\hline $\begin{array}{l}\text { Inter-apical area with/without } \\
\text { satellite }\end{array}$ & $\begin{array}{l}\text { Without } \\
\text { satellite } \\
\text { (rarely with } \\
\text { few satellites) }\end{array}$ & $\begin{array}{l}\text { Without } \\
\text { satellite } \\
\text { (rarely with } \\
\text { few satellites) }\end{array}$ & $\begin{array}{l}\text { Without } \\
\text { satellite } \\
\text { (rarely with } \\
\text { few satellites) }\end{array}$ & $\begin{array}{l}\text { With } \\
\text { satellite }\end{array}$ & $\begin{array}{l}\text { With } \\
\text { satellite }\end{array}$ & $\begin{array}{l}\text { With } \\
\text { satellite }\end{array}$ \\
\hline
\end{tabular}

These morphotypes are taxon-specific, and this pattern of distinct attributes among the different species under different genera remains ill-defined, as predicted by Stevanato et al. (2019), Whereas Stevanato et al. (2019) used leaf samples of different species of Cyperaceae, the present study used not only leaf blades but also leaf sheaths and culms. However, remarkable variations were not found in different parts of the same species, although interspecific variation was prominent. Recently, Murungi and Bamford (2020) emphasized the morphology, surface texture, and ornamentation of achene cones (conical in the present study) as a taxonomic characteristic rather than the size of the morphotypes. 


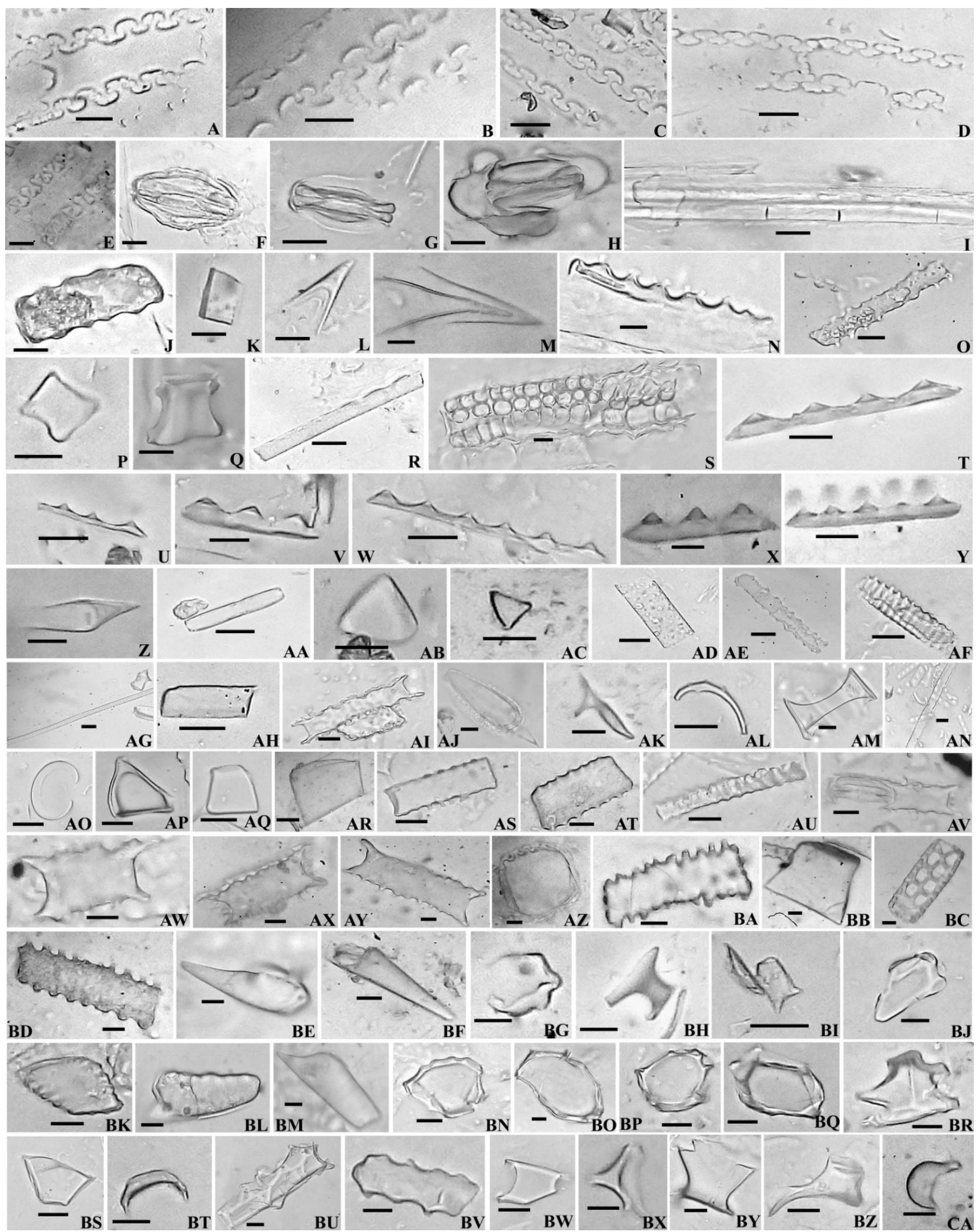

Figure 1. Phytolith morphotypes observed in the studied species of Carex. A. Elongate bulbous margin in Carex cruciata (lb), B. Elongate bulbous margin in C. cruciata (1s), C. Elongate bulbous margin in Carex filicina (lb), D. Elongate bulbous margin in C. filicina (ls), E. Elongate bulbous margin in Carex setigera (lb), F. Stomatal complex in C. cruciata (lb), G. Stomatal complex in C. filicina (lb), H. Stomatal complex in C. setigera (lb), I. Elongate articulated in C. cruciata (lb), J. Trapezoid sinuate in C. cruciata (lb), K. Trapezoid in C. cruciata (ls), L. Lanceolate psilate in C. cruciata (lb), C. filicina (lb), M. Lanceolate reflexed in C. setigera (lb), N. Elongate 
papillate in C. cruciata (lb), O. Elongate papillate in C. cruciata (ls), P. Rondel tenuis reflexed in C. cruciata (lb), Q. Rondel tenuis reflexed in C. filicina (ls), R. Elongate psilate in C. cruciata (lb), S. Favose in C. cruciata (lb), C. filicina (lb), T. Conical in C. cruciata (lb), U. Conical in C. cruciata (ls), V. Conical in C. filicina (lb), W. Conical in C. filicina (ls), X. Conical in C. setigera (lb), Y. Conical in $C$. setigera (ls), Z. Elongate lanceolate in C. cruciata (ls), AA. Rectangle psilate in C. cruciata (ls), AB. Scutiform triangular in $C$. cruciata (ls), AC. Scutiform triangular in C. setigera (ls), AD. Tabular scrobiculate in C. cruciata (cu), AE. Elongate sinuate in $C$. cruciata (cu), AF. Tracheary in C. cruciata (cu), AG. Tracheary in C. filicina (lb), C. setigera (lb), AH. Tabular psilate in C. cruciata (cu), AI. Elongate concave with verrucate in C. filicina (lb), AJ. Acute bulbosus in C. filicina (lb), AK. Trichome in C. filicina (lb), AL. Crescenti in C. filicina (ls), AM. Tabular gibbous in C. filicina (ls), AN. Elongate echinate in C. filicina (ls), AO. Crescenti convex in $C$. filicina (ls), AP. Cuneiform in C. filicina (ls), AQ. Tower in C. filicina (ls), AR. Tower in C. setigera (ls), AS. Tabular sinuate in $C$. filicina $(\mathrm{Cu})$, AT. Tabular sinuate in $C$. setigera $(\mathrm{lb})$, C. setigera (ls), AU. Elongate laminate in C. filicina (cu), C. setigera (cu), AV. Tabular concave echinate with stomata in $C$. setigera (lb), AW. Tabular concave echinate in $C$. setigera (lb), AX. Tabular concave with verrucate in C. cruciata (lb), C. filicina (ls), C. setigera (lb), AY. Tabular concave in C. setigera (lb), AZ. Semi square sinuate in $C$. setigera (lb), BA. Tabular columellate in $C$. setigera (lb), BB. Tower wide in $C$. setigera (lb), BC. Tabular favose castelate in $C$. setigera (ls), BD. Tabular crenate in $C$. setigera $(\mathrm{ls}), \mathrm{BE}$. Clavate in $C$. setigera $(\mathrm{cu}), \mathrm{BF}$. Acuminate hollow in $C$. setigera $(\mathrm{cu}), \mathrm{BG}$. Unclassified in C. cruciata (lb), BH. Unclassified in C. cruciata (lb), BI. Unclassified in C. cruciata (ls), BJ. Unclassified in C. cruciata (cu), BK. Unclassified in C. cruciata (cu), BL. Unclassified in C. filicina (lb), BM. Unclassified in C. setigera (lb), BN. Unclassified in C. cruciata (cu), BO. Unclassified in C. filicina (lb), BP. Unclassified in C. filicina (cu), BQ. Unclassified in C. setigera (lb), BR. Unclassified in C. cruciata (cu), BS. Unclassified in C. filicina (lb), BT. Unclassified in C. filicina (lb), BU. Unclassified in C. filicina (lb), BV. Unclassified in C. filicina (lb), BW. Unclassified in C. filicina (lb), BX. Unclassified in C. filicina (lb), BY. Unclassified in C. filicina (lb), BZ. Unclassified in C. filicina (cu), CA. Unclassified in C. setigera (lb), (lb=leaf blade, ls=leaf sheath, cu=culm; scale bar $=10 \mu \mathrm{m})$.

According to Fernández Honaine et al. (2009), a polygonal shape of the conical base was observed in the fruit of the species of both Carex and Cyperus, but in presently investigated species of Carex and Cyperus, there is no evidence of such a polygonal shape of conical base morphotypes, because only vegetative samples were analyzed.

Among conical second descriptors, the number of peripheral satellites, length of the conical, height of the conical, height of the apex, the width of the apex, and interapical area distance were also measured (Figures 5 and 6; Table S1), Of the quantitative characteristics, the interapical area distance (Figure 5.A-B) is an important characteristic that was previously suggested by Ollendorf (1992), and it is also applicable for the studied species. Here, the inter-apical area was without satellites (or rarely with satellites), and the inter-apical distance in the Carex species was more than $4 \mu \mathrm{m}$ (Figures 3, 6), Meanwhile, in Cyperus species, the inter-apical area had distinct satellites, and the distance was less than $4 \mu \mathrm{m}$ (Figures 3, 6), Fernández Honaine et al. (2009) and Stevanato et al. (2019) considered the length of the conical to be a significant character. Murungi (2017) illustrated leaf cone morphotypes of three species of Cyperus (Cyperus congestus, C. haematocephalus, and C. semitrifidus), However, these qualitative and quantitative characteristics were not considered.

From their quantitative and qualitative analysis of nine South African Cyperus species, Murungi (2017) and Murungi and Bamford (2020) concluded that cone phytoliths were found in most species of Cyperaceae but were not the dominant morphotype. They also inferred that the variation in leaf cones in terms of size and presence of satellites are generally less important taxonomic characteristics for the family Cyperaceae. In addition, Murungi and Bamford (2020) did not find any genusspecific (Cyperus type) phytoliths in Cyperus. However, they did not evaluate any of the species of Carex s.s. in their studies. The current study reports a considerable difference between the conical phytolith structures, based on which we can distinguish the studied genera.

Furthermore, Murungi and Bamford (2020) observed that achene cone phytoliths are not always cone-shaped in lateral view (i.e., they may be polygonal or isodiametric or sometimes elongate in structure), and leaf cone features are not consistent within a single genus, indicating its taxonomic applicability. The current study also supports this finding. Other morphotypes, such as stomatal complexes and tabular and/or blocky parallelepiped morphotypes, are the most dominant (Murungi and Bamford 2020), In the present work, stomatal complexes and tabular morphotypes were also reported.

In recent phylogenetic studies on different taxa of monocotyledons, the presence and absence of different phytolith morphotypes have been considered for taxonomic treatment (Prychid et al. 2004), Variations of morphotypes or sub-morphotypes were not considered for particular taxon delimitation. But this study shows that the value of conical length for Carex and Cyperus species is also important. Other important characteristics are the height of the apex (Stevanato et al. 2019), the height of the conical, and the width of the apex (for the first time reported in this study), However, from a taxonomic point of view, the qualitative characteristics of phytolith morphotypes show more notable value than the quantitative characteristics.

This study demonstrates that the presence or absence of some phytolith morphotypes (e.g., elongate bulbous margin, Figure 1.A-E; lanceolate psilate, Figure 1.L; tabular concave with verrucate, Figure 1.AX; tabular concave columellate, Figure 2.B; ovate granulate, Figure 2.Q; and orbicular concave, Figure 2.AP) are genusspecific and can provide further support for taxonomists to confirm morphological and phylogenetic classifications of the genera in Cyperaceae, as stated by Murungi (2017). 


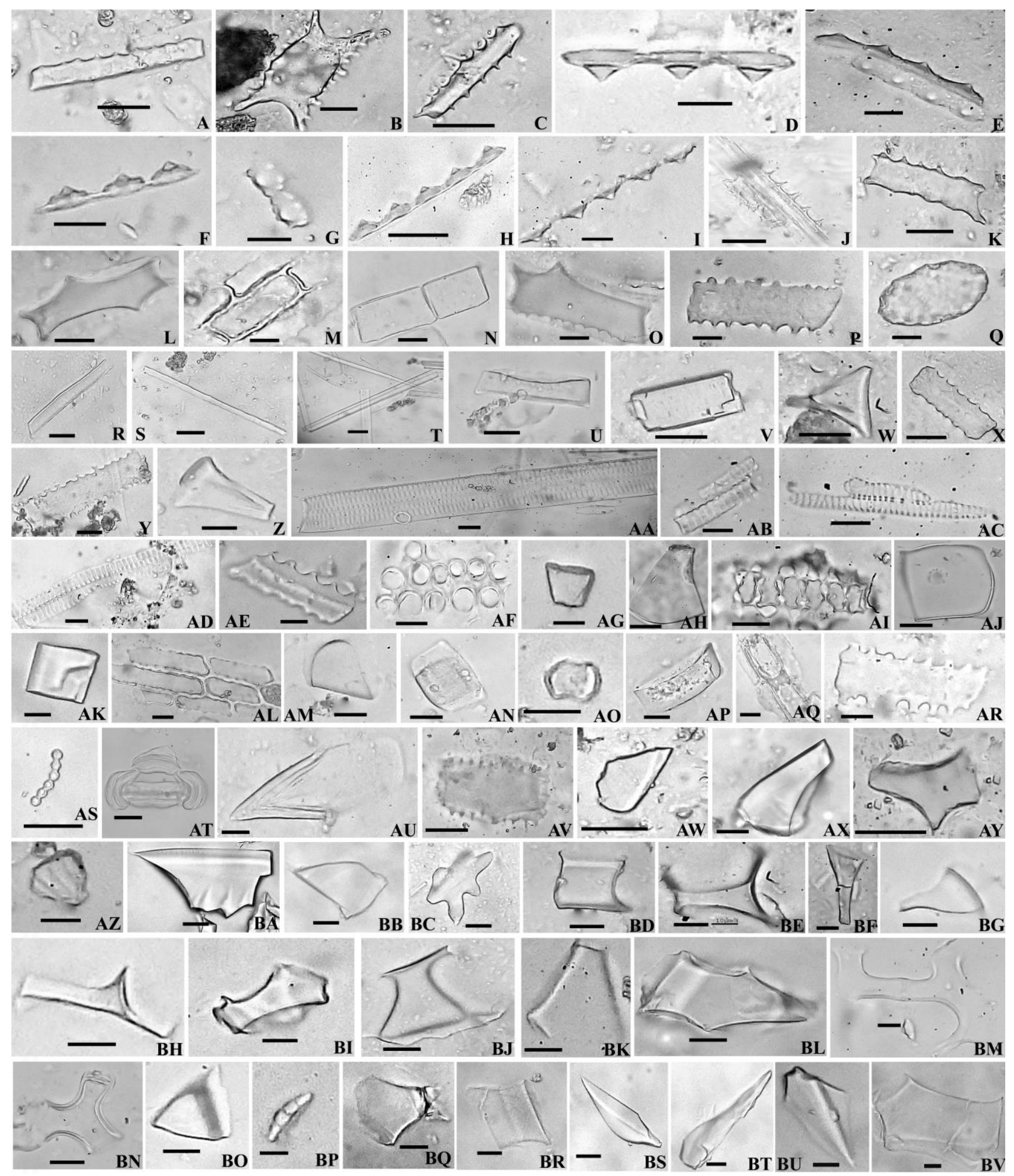

Figure 2. Phytolith morphotypes observed in the studied species of Cyperus. A. Elongate sinuate in Cyperus distans (lb), B. Tabular concave columellate in C. distans (lb), C. imbricatus (lb), C. Elongate echinate in C. distans (lb), D. Conical in C. distans (lb), E. Conical in C. distans (ls), F. Conical in C. exaltatus. (lb), G. Conical in C. exaltatus (ls), H. Conical in C. imbricatus (lb), I. Conical in C. imbricatus (ls), J. Elongate acute in $C$. distans $(\mathrm{lb}), \mathrm{K}$. Tabular concave echinate in $C$. distans (lb), L. Tabular concave acute in $C$. distans (lb), M. Tabular in C. distans (lb), N. Tabular in C. distans (cu), C. exaltatus (cu), O. Tabular papillate in C. distans (lb), P. Tabular papillate in C. exaltatus (lb), Q. Ovate granulate in C. distans (lb), C. exaltatus (lb), C. imbricatus (ls), R. Elongate psilate in C. distans (lb), S. Elongate psilate in C. distans (ls), C. imbricatus (ls), T. Elongate psilate in C. distans (cu), C. imbricatus (cu), U. Elongate psilate in C. exaltatus (lb), V. Tabular rectangular in C. distans (lb), W. Scutiform triangular in C. distans (ls), X. Tabular crenate in C. distans (ls), Y. Tabular crenate in C. imbricatus (lb), Z. Tower in C. distans (ls), AA. Tracheary in C. distans (cu), AB. Tracheary in C. exaltatus (lb), AC. Tracheary in C. exaltatus (cu), AD. Tracheary in C. imbricatus (cu), AE. Tabular echinate verrucate in C. exaltatus (lb), AF. Favose in C. exaltatus (lb), AG. Tower wide in C. exaltatus (lb), AH. Tower wide in C. imbricatus (ls), AI. 
Elongate sulcate in C. exaltatus (lb), AJ. Square in C. exaltatus (lb), AK. Square in C. imbricatus (cu), AL. Tabular sinuate in $C$. exaltatus (lb), AM. Cuneiform in C. exaltatus (lb), AN. Tabular scrobiculate in C. exaltatus (lb), AO. Short saddle in C. exaltatus (lb), AP. Orbicular concave in C. distans (cu), C. exaltatus (cu), C. imbricatus (cu), AQ. Bulliform parallelepipedal articulated in C. exaltatus (cu), AR. Tabular pilate in C. imbricatus (lb), AS. Polylobate in C. imbricatus (lb), AT. Stomatal complex in C. imbricatus (lb), AU. Unclassified in $C$. distans (lb), AV. Unclassified in C. distans (lb), AW. Unclassified in $C$. distans (lb), AX. Unclassified in $C$. distans (lb), AY. Unclassified in C. distans (ls), AZ. Unclassified in C. exaltatus (lb), BA. Unclassified in C. imbricatus(lb), BB. Unclassified in C. imbricatus (ls), BC. Unclassified in $C$. distans (1s), BD. Unclassified in $C$. distans (1s), BE. Unclassified in $C$. distans (ls), BF. Unclassified in C. distans (1s), BG. Unclassified in C. exaltatus (lb), BH. Unclassified in C. exaltatus (ls), BI. Unclassified C. imbricatus (cu), BJ. Unclassified in C. distans (ls), BK. Unclassified C. imbricatus (lb), BL. Unclassified in C. distans (ls), BM. Unclassified $C$. exaltatus (lb), BN. Unclassified C. exaltatus (ls), BO. Unclassified C. exaltatus (ls), BP. Unclassified C. imbricatus (lb), BQ. Unclassified C. imbricatus (lb), BR. Unclassified C. imbricatus (lb), BS. Unclassified C. imbricatus (lb), BT. Unclassified C. imbricatus (lb), BU. Unclassified C. imbricatus (lb), BV. Unclassified C. imbricatus (ls), (lb=leaf blade, ls=leaf sheath, cu=culm; scale bar=10 $\mu \mathrm{m}$ )
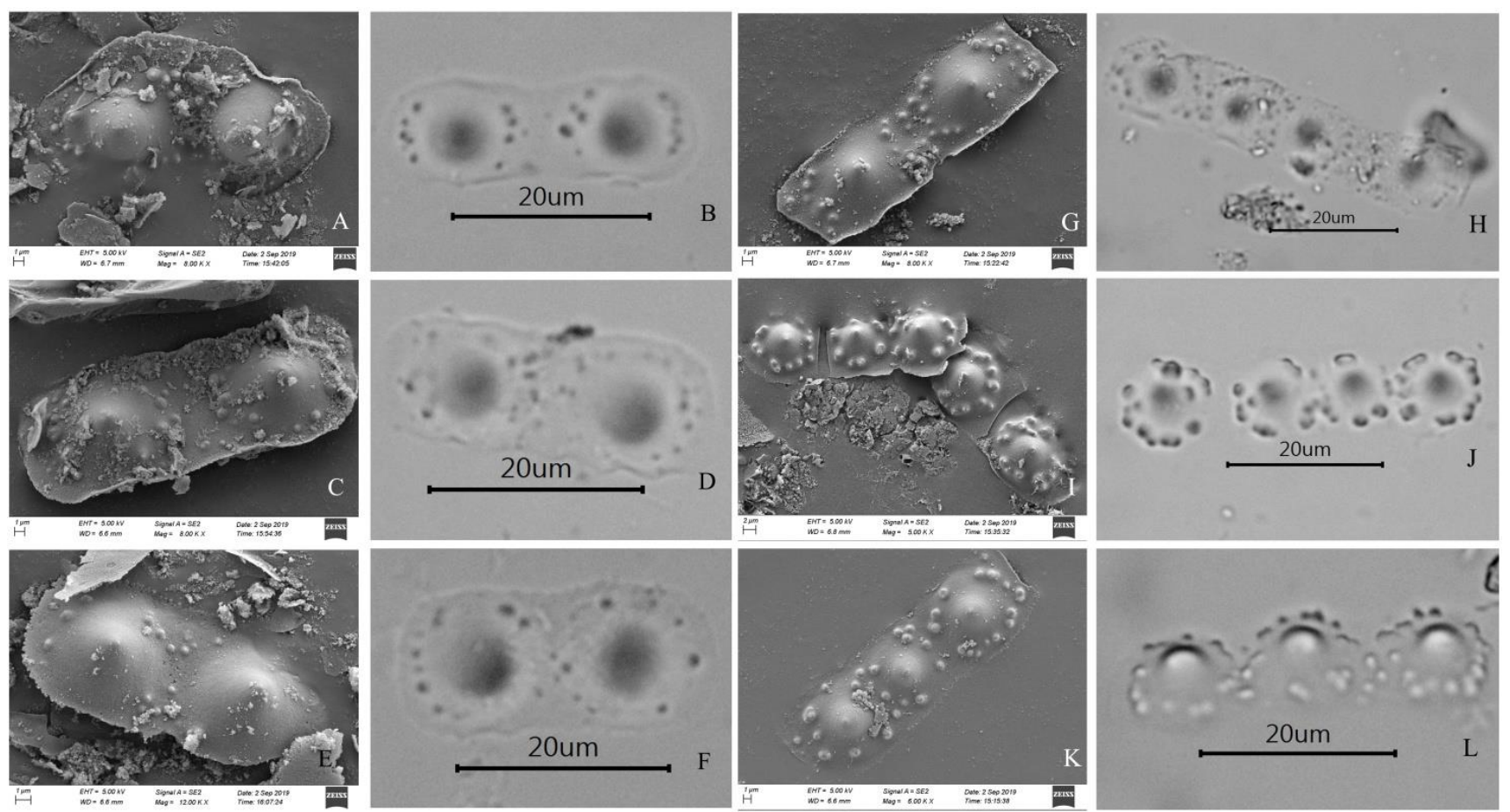

Figure 3. FE-SEM and LM images of conical morphotype and arrangement of satellites in the studied species of Carex and Cyperus. A, B. Carex cruciata, C, D. Carex filicina, E, F. Carex setigera, G, H. Cyperus distans, I, J. Cyperus exaltatus, K, L. Cyperus imbricatus.
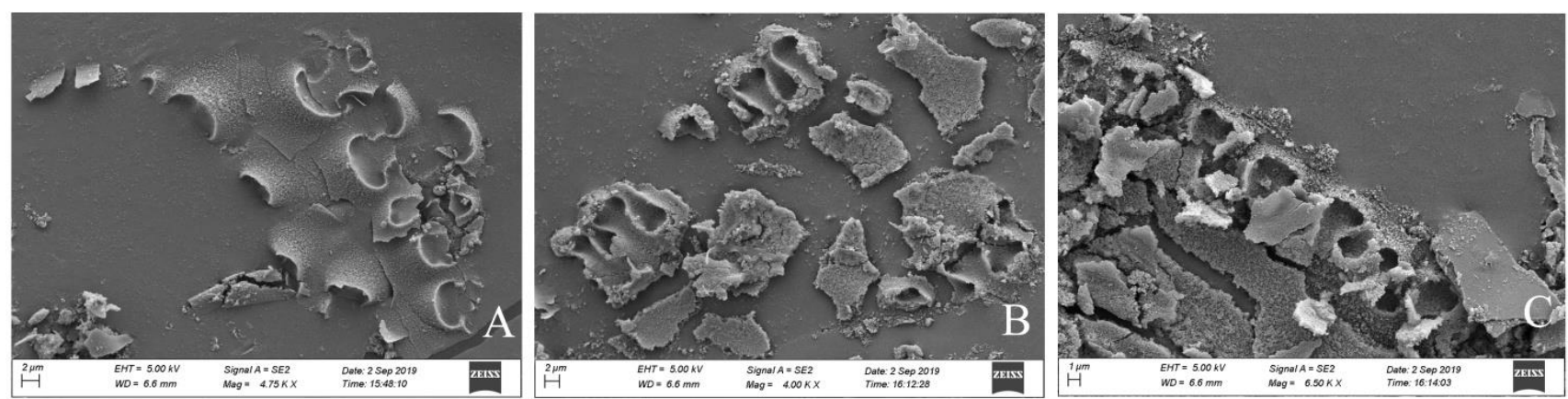

Figure 4. FE-SEM images of elongate bulbous margin phytolith morphotypes in the studied species of Carex. A. Carex cruciata, B. Carex filicina, C. Carex setigera 

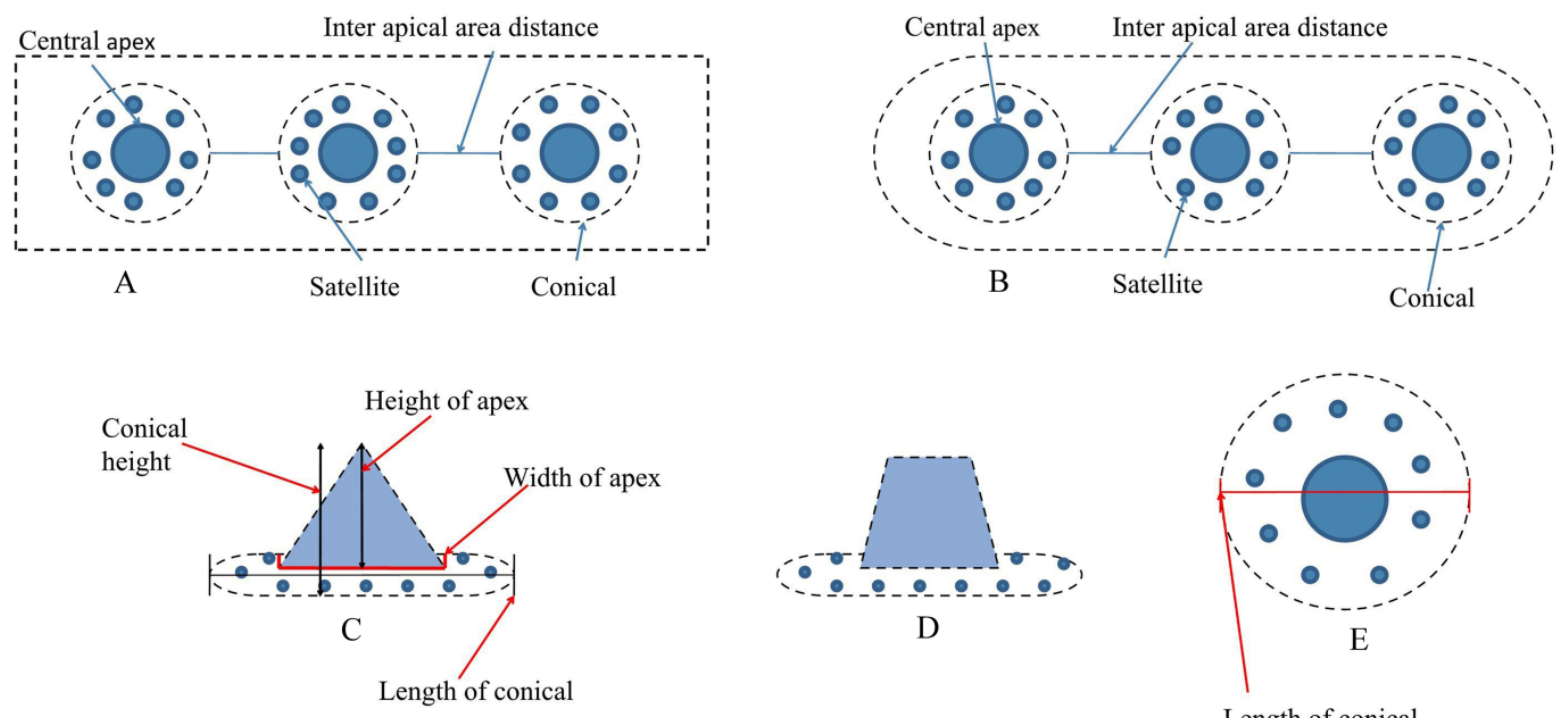

Length of conical

Figure 5. Diagrammatic representation and characterization of different conical morphotypes. A. Square shape of conical. B. Oblong shape of conical. C. Acuminate type of conical and different measurement area of conical (length of conical, height of conical, height of apex, width of apex), D. Blunt type of conical. E. Arrangement form of satellite and measurement area for length of conical (following Stevanato et al. 2019)

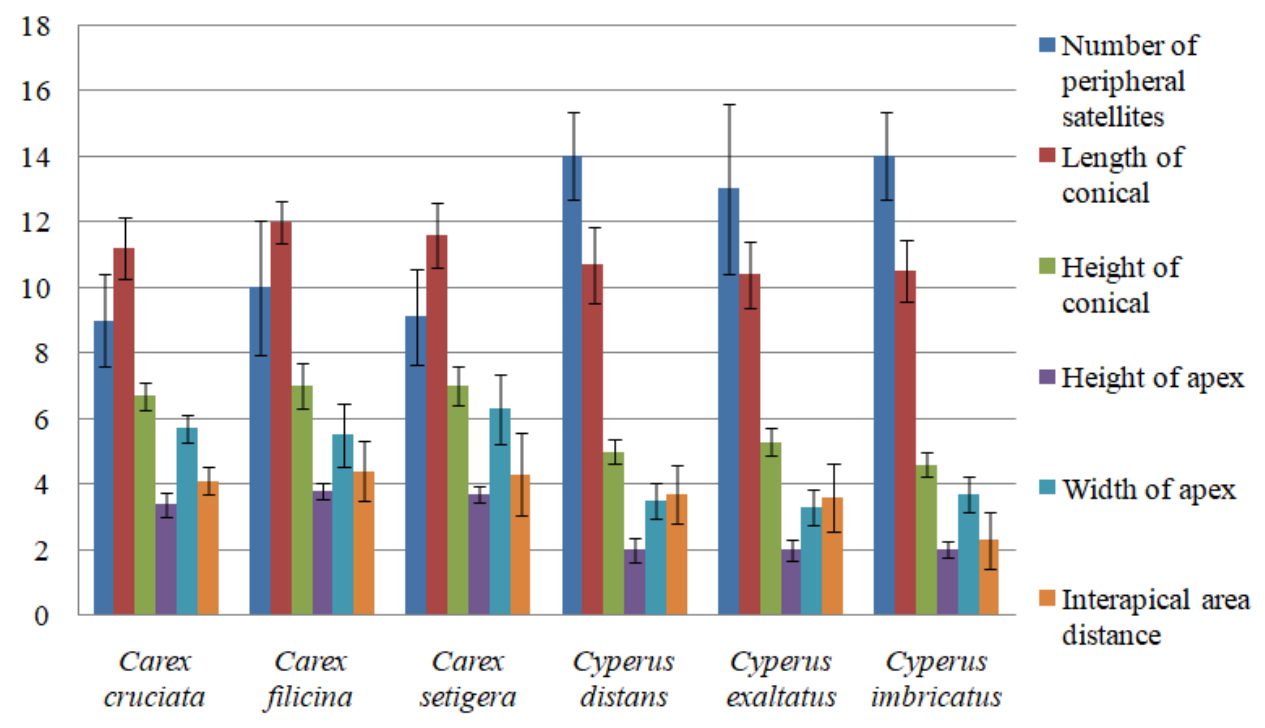

Figure 6. Bar graph comparing the quantitative data of conical morphotypes in the studied species of Carex and Cyperus (data are presented in Table S1)

Table S1. Measurements of different quantitative characters of conical in studied species of Carex and Cyperus

\begin{tabular}{|c|c|c|c|c|c|c|}
\hline Conical characters & $\begin{array}{c}\text { Carex cruciata } \\
\text { Value }=\text { Mean } \pm \text { SD }\end{array}$ & $\begin{array}{c}\text { Carex filicina } \\
\text { Value }=\text { Mean } \pm \text { SD }\end{array}$ & $\begin{array}{c}\text { Carex setigera } \\
\text { YValue }=\text { Mean } \pm \text { SD }\end{array}$ & $\begin{array}{c}\text { Cyperus distans } \\
\text { Value }=\text { Mean } \pm \text { SD }\end{array}$ & $\begin{array}{l}\text { Cyperus exal } \\
\text { Value }=\text { Mean }\end{array}$ & $\begin{array}{l}\text { is Cyperus imbricatus } \\
\text { D)(Value }=\text { Mean } \pm \text { SD })\end{array}$ \\
\hline Number of peripheral satellite & $9 \pm 1.4$ & $10 \pm 2.04$ & $9.1 \pm 1.46$ & $14 \pm 1.33$ & $13 \pm 2.61$ & $14 \pm 1.33$ \\
\hline Length of conical $(\mu \mathrm{m})$ & $11.2 \pm 0.92$ & $12 \pm 0.64$ & $11.6 \pm 1$ & $10.7 \pm 1.17$ & $10.4 \pm 1.01$ & $10.5 \pm 0.94$ \\
\hline Height of conical $(\mu \mathrm{m})$ & $6.7 \pm 0.42$ & $7 \pm 0.69$ & $7 \pm 0.58$ & $5 \pm 0.37$ & $5.3 \pm 0.43$ & $4.6 \pm 0.36$ \\
\hline Height of apex $(\mu \mathrm{m})$ & $3.4 \pm 0.37$ & $3.8 \pm 0.27$ & $3.7 \pm 0.26$ & $2 \pm 0.37$ & $2 \pm 0.30$ & $2 \pm 0.25$ \\
\hline Width of apex $(\mu \mathrm{m})$ & $5.7 \pm 0.40$ & $5.5 \pm 0.97$ & $6.3 \pm 1.07$ & $3.5 \pm 0.54$ & $3.3 \pm 0.56$ & $3.7 \pm 0.54$ \\
\hline Interapical area distance $(\mu \mathrm{m})$ & $4.1 \pm 0.42$ & $4.4 \pm 0.92$ & $4.3 \pm 1.26$ & $3.7 \pm 0.87$ & $3.6 \pm 1.02$ & $2.3 \pm 0.86$ \\
\hline
\end{tabular}

Note: Calculation was executed separately for each of the conical characters; total $n=80$ 
In conclusion, The present investigation describes the phytolith morphotypes in six species of Cyperaceae (under two genera), and some distinct morphotypes are found for the genera Carex and Cyperus. Of all the studied morphotypes, the conical morphotype has important characteristics with taxonomic applicability. In Carex $(C$. cruciata, C. filicina, C. setigera), elongate bulbous margin, lanceolate psilate, and tabular concave verrucate type of phytolith were commonly found, whereas, in Cyperus (C. distans, C. exaltatus, C. imbricatus), tabular concave columellate, ovate granulate, and orbicular concave morphotypes were constantly present. Both qualitative and quantitative data are helpful in the study of patterns and differentiation of phytolith morphotypes among the studied species of Cyperaceae, although quantitative data are more useful in the analysis of conical morphotypes while qualitative data are more useful for other morphotypes. As some morphotypes are sometimes phenotypically plastic, common to other botanical families, further extensions of this work covering a considerable number of species would help in the identification of species and infraspecific taxa of Cyperaceae. From these data, it can be concluded that some of the morphotypes are found throughout the studied species, and some are constant for the genera. Thus, some morphological differences, such as the height, length, or inter-apical area of conical phytoliths, could be important for the identification of Cyperaceous taxa. However, a detailed study of the phytolith morphotypes in other taxa of Cyperaceae is needed for species and infra-specific identification.

\section{ACKNOWLEDGEMENTS}

The first author is grateful to the Government of West Bengal, India for providing the Swami Vivekananda Merit Cum Means Scholarship for the work. The authors are also grateful to University Science and Instrumentation Center (USIC) and the Head(s) of the Department of Botany, The University of Burdwan, India for providing essential instrumental and laboratory facilities. The authors also thank the Director, Botanical Survey of India (BSI) and Head of the Office and Scientist-E, CNH, Howrah for providing permission for the CAL herbarium consultation.

\section{REFERENCES}

Arisdason W, Lakshminarasimhan P. 12 October 2017 onward. ENVIS Centre on Floral Diversity. Central National Herbarium, Botanical Survey of India, Howrah. http://www.bsienvis.nic.in/Database/Status of Plant Diversity in India 17566.aspx

Biswas O, Ghosh R, Paruya DK, Mukherjee B, Thapa KK, Bera S. 2016. Can grass phytoliths and indices be relied on during vegetation and climate interpretations in the Eastern Himalayas? Studies from Darjeeling and Arunachal Pradesh, India. Quat Sci Rev 134: 114-132. DOI: 10.1016/j.quascirev.2016.01.003

Bobrov AA, Semenov AN, Alexeev YuE. 2016. Phytoliths of species some genera of the family Cyperaceae. Environmental Dynamics and Global Climate Change 7 (1): 27-33. DOI: 10.17816/edgec7127-33

Browning J, Gordon Gray KD. 1995. Studies in Cyperaceae in southern Africa, 26: Glume epidermal silica deposits as a character in generic delimitation of Costularia and Cyathocoma as distinct from Tetraria and other allies. S Afr J Bot 61: 66-71. DOI: 10.1016/S02546299(15)30481-6

Carnelli AL, Theurillat JP, Madella M. 2004. Phytolith types and typefrequencies in subalpine-alpine plants species of the Europian Alps. Rev Palaeobot Palyno 129: 39-65. DOI: 10.1016/j.revpalbo.2003.11.002

Collura LV, Neumann K. 2017. Wood and bark phytoliths of West African woody plants. Quat Intl 434: 142-159. DOI: 10.1016/j.quaint.2015.12.070

Dhooge S. 2005. Systematic revision and phylogeny of the Andean scirpoids (Cyperaceae, Scirpeae), Universiteit Gent, Gent, Belgium.

Ebigwai JK, Nyannayo BL, Egbe AE, Asuquo E, Aniekebo IH. 2015. Variation Of Phytolith Morphotypes Of Some Members Of Cucurbitaceae Juss. J Pharm Biol Sci 10 (6): 100-115 DOI: 10.9790/3008-1062100115

Eksambekar S. 2009. Review of Phytolith Research: Scope and Applications. Korean J Quat Res 23 (2): 1-12.

Fahmy AG. 2008. Diversity of lobate phytoliths in grass leaves from the Sahel region, West Tropical Africa: Tribe Paniceae. Plant Syst Evol 270: 1-23. DOI: 10.1007/s00606-007-0597-z

Fernández Honaine M, Borrelli NL, Osterrieth M, Del Rio LS. 2013. Amorphous silica biomineralizations in Schoenoplectus californicus (Cyperaceae): their relation with maturation stage and silica availability. Bol Soc Argent Bot 48: 247-259. DOI: 10.31055/1851. 2372.v48.n2.6208

Fernández Honaine M, Zucol AF, Osterrieth ML. 2009. Phytolith analysis of Cyperaceae from the Pampean region, Argentina. Aust J Bot 57: 512-523. DOI: $10.1071 / \mathrm{bt} 09041$

Gallego L, Distel RA. 2004. Phytolith Assemblages in Grasses Native to Central Argentina. Ann Bot 94: 865-874. DOI: 10.1093/aob/mch214

Hodson MJ, White PJ, Mead A, Broadley MR. 2005. Phylogenetic variation in the silicon composition of plants. Ann Bot 96: 10271046. DOI: $10.1093 / \mathrm{aob} / \mathrm{mci} 255$

Houyouan L, Naiqin W, Baozhu L. 1997. Recognition of rice phytoliths. In: Pinilla A, Juan-Tressaras J, Machado MJ (eds) The State of the Art of Phytoliths in Soils and Plants. Springer, Madrid.

Karthikeyan S, Jain SK, Nayar MP, Sanjappa M. 1989. Florae Indicae Enumeratio: Monocotyledinae. (Flora of India), Botanical Survey of India, Calcutta.

Kondo R, Childs CW, Atkinson IAE, Pritchard T. 1994. Opal Phytoliths of New Zealand. Manaaki Whenua Press, Lincoln, New Zealand.

Lisztes-Szabo Z, Kovacs S, Balogh P, Daroczi L, Penksza K, Peto A. 2015. Quantifiable differences between phytolith assemblages detected at species level: analysis of the leaves of nine Poa species (Poaceae), Acta Soc Bot Pol 84 (3): 369-383. DOI: 10.5586/asbp.2015.027

Lisztes-Szabo Z, Kovacs S, Peto A. 2014. Phytolith analysis of Poa pratensis (Poaceae) leaves. Turk J Bot 38: 851-863. DOI: 10.3906/bot-1311-8

Lu H, Liu K. 2003. Phytoliths of common grasses in the coastal environments of southeastern USA. Estuar Coast Shelf Sci 58: 587 600. DOI: 10.1016/S0272-7714(03)00137-9

Madella M, Alexandre A, Ball T. 2005. International Code for Phytolith Nomenclature 1.0. Ann Bot 96: 253-260. DOI: 10.1093/aob/mci172

McNamee C. 2013. Soil Phytolith Assemblages of the American South West: The Use of Historical Ecology in Taphonomic Studies. Department of Archaeology, University of Calgary, Calgary, Alberta DOI: $10.11575 /$ PRISM/27822

Mehra PN, Sharma OP. 1965. Epidermal silica cells in the Cyperaceae. Botan Gaz 126 (1): 53-58.

Mercader J, Astudillo F, Barkworth M, Bennett T, Esselmont C, Kinyanjui R, Grossman DL, Simpson S, Walde D. 2010. Poaceae Phytoliths from the Niassa Rift, Mozambique. J Archaeol Sci 37: 1953-1967. DOI: $10.1016 /$ j.jas.2010.03.001

Metcalfe CR. 1971. Anatomy of the Monocotyledons V. Cyperaceae. Clarendon Press, Oxford.

Muasya AM, Simpson DA, Verboom GA, Goetghebeur P, Naczi RFC, Chase MW, Smets E. 2009. Phylogeny of Cyperaceae based on DNA sequence data: current progress and future prospects. Bot Rev 75: $2-$ 21. DOI: $10.1007 / \mathrm{s} 12229-008-9019-3$

Mudassir AB, Shakoor SA, Badgal P, Soodan AS. 2018. Taxonomic Demarcation of Setaria pumila (Poir.) Roem. \& Schult., S. verticillata (L.) P. Beauv. and S. viridis (L.) P. Beauv. (Cenchrinae, Paniceae, 
Panicoideae, Poaceae) from Phytolith Signatures. Front Plant Sci 9(864): 1-32. DOI: 10.3389/fpls.2018.00864

Mulholland SC, Ollendorf AL, Rapp Jr G. 1989. Maize phytolith assemblages: Data from the Northern Plains, USA.

Murungi ML, Bamford MK. 2020. Revised taxonomic interpretations of Cyperaceae phytoliths for (paleo)botanical studies with some notes on terminology. Rev Palaeobot Palyno 275 (104): 189. DOI: 10.1016/ j. revpalbo.2020.104189.

Murungi ML. 2017. Phytoliths at Sibudu (South Africa): Implications for Vegetation, Climate and Human Occupation During the MSA. School of Geosciences and the Evolutionary Studies Institute. [Thesis]. The University of the Witwatersrand, South Africa.

Naskar M, Bera S. 2018. Taxonomic assessment of opal phytoliths from grasses of deltaic West Bengal, India. Nord J Bot 36 (4): 1-42. DOI: 10.1111/njb.01695

Netolitzky F. 1929. Die Kieselkorper: Die Kalksalze als Zellinhaltskorper. Handbuch der Pflanzenanatomie. Berlin. [Germany]

Neumann K, Strömberg CAE, Ball T, Albert RM, Vrydaghs L, Cummings LS. 2019. International Code for Phytolith Nomenclature (ICPN) 2.0. Ann Bot 124 (2): 189-199. DOI: 10.1093/aob/mcz064

Ollendorf AL. 1992. Toward a Classification Scheme of Sedge (Cyperaceae) Phytoliths. pp. 91-111. In: Rapp Jr. G, Mulholland SC (eds) Phytolith Systematics: Emerging Issues, 1st ed. Plenum Press, New York.

Pal H, Choudhury MD. 2014. Taxonomic Status of Cyperaceae of Southern Assam. Pleione 8 (1): 149-157.

Pearsall DM, Piperno DR, Dinan EH, Umlauf R, Zhao Z, Benfer RA. 1995. Distinguishing rice (Oryza Sativa Poaceae) from wild Oryza species through phytolith analysis - Results of preliminary research. Econ Bot 49: 183-196. DOI: 10.1007/BF02862923

Piperno DR, Pearsall DM. 1998. The silica bodies of tropical American grasses: morphology, taxonomy and implications for grass systematics and fossil phytolith identification. Smithsonian Contrib Bot 85: 1-40. DOI: 10.5479/si.0081024X.85

Piperno DR. 1985. Phytolith analysis and tropical paleo-ecology: Production and taxonomic significance of siliceous forms in new world plant domesticates and wild species. Rev Palaeobot Palyno 45: 185-228. DOI: 10.1016/0034-6667(85)90002-8

Piperno DR. 1988. An Archaeological and Geological Perspective. Academic Press, San Diego.

Piperno DR. 2006. Phytoliths: A Comprehensive Guide for Archaeologists and Paleoecologists. Altamira Press, Oxford.

Prasad VP, Singh NP. 2002. Sedges of Karnataka. Scientific Publisher, Jodhpur, India.

Prychid CJ, Rudall PJ, Gregory M. 2004. Systematics and biology of silica bodies in monocotyledons. Bot Rev 69 (4): 377-440. DOI: 10.1663/0006-8101(2004)069[0377: SABOSB]2.0.CO;2
Reznicek AA, Catling PM. 1986. Vegetative shoots in the taxonomy of sedges (Carex, Cyperaceae), Taxon 35(3): 495-50. DOI: $10.2307 / 1221903$

Reznicek AA. 1990. Evolution in sedges (Carex, Cyperaceae), Can J Bot 68: 1409-1432. DOI: 10.1139/b90-180

Rosen AM. 1992. Preliminary Identification of Silica Skeletons from Near Eastern Archaeological Sites: An anatomical approach. In: Rapp Jr G, Mulholland SC (eds.), Phytolith Systematics: Advances in Archaeological and Museum Science. New York, Plenum Press.

Shakoor SA, Soodan AS, Kumar K. 2014. Morphological diversity and frequency of phytolith types in gaint reed Arundo donax (L.), World Appl Sci J 29 (7): 926-932. DOI: 10.5829/idosi.wasj.2014.29.07.14

Simpson DA, Inglis CA. 2001. Cyperaceae of economic, ethnobotanical and horticultural importance: a checklist. Kew Bull 56: 257-360. DOI: $10.2307 / 4110962$.

Starr JR, Bayer RJ, Ford BA. 1999. The phylogenetic position of Carex section Phyllostachys and its implications for phylogeny and subgeneric circumscription in Carex (Cyperaceae), Am J Bot 86 (4): 563-577. DOI: $10.2307 / 2656818$.

Starr JR, Ford BA. 2001. The taxonomic and phylogenetic utility of vegetative anatomy and fruit epidermal silica bodies in Carex section Phyllostachys (Cyperaceae), Can J Bot 79: 362-379. DOI: 10.1139/b01-004.

Stevanato M, Rasbold GG, Parolin M, Luz LD, Lo E, Weber P, Trevisan R, Caxambu MG. 2019. New characteristics of the papillae phytolith morphotype recovered from eleven genera of Cyperaceae. Flora 253: 49-55. DOI: $10.1016 /$ j.flora.2019.03.012

Thiers B. 2019 [continuously updated]. Index Herbariorum: A global directory of public herbaria and associated staff. New York Botanical Garden's Virtual Herbarium. http://sweetgum.nybg.org/science/ih/

Toivonen H, Timonen T. 1976. Perigynium and achene epidermis in some species of Carex, subg. Vignea (Cyperaceae), studied by scanning electron microscopy. Ann Bot Fenn. 13: 49-59.

Twiss PC, Suess E, Smith RM. 1969. Morphological Classification of Grass Phytoliths. Soil Sci So Am Proc 33 (1): 109-115.

Wallis L. 2003. An overview of leaf phytolith production patterns in selected northwest Australian flora. Rev Palaeobot Palyno 125: 201248. DOI: $10.1016 / \mathrm{S} 0034-6667(03) 00003-4$

Wang YJ, Lu HY. 1993. The Study of Phytolith and its Application. China Ocean press, Beijing.

WCSP (2020), World Checklist of Selected Plant Families. Facilitated by the Royal Botanic Gardens, Kew. http://wcsp.science.kew.org/

Zhao Z, Pearsall DM, Benfer RA. Piperno DR. 1998. Distinguishing rice (Oryza sativa Poaceae) from wild Oryza species through phytolith analysis, II: Finalized method. Econ Bot 52: 134-145. DOI: 10.1007/BF02861201 